\title{
The Evaluation of Progesterone and Dysmenorrhea Levels after the Manual Therapy in Young Women in Relation to the Use of Non-Steroidal Anti-Inflammatory Drugs
}

\author{
Zofia Barcikowska1*, Magdalena Emilia Grzybowska², Elżbieta Rajkowska-Labon³, Rita Hansdorfer-Korzon ${ }^{4}$, \\ Katarzyna Zorena ${ }^{5}$ \\ 1 Department of Immunobiology and Environment Microbiology, Medical University of Gdańsk, Dębinki 7, \\ 80-211 Gdańsk, Poland, email: zofia.barcikowska@gumed.edu.pl (Z.B.) \\ 2 Department of Gynecology, Gynecologic Oncology and Gynecologic Endocrinology, Medical University of \\ Gdańsk, Smoluchowskiego 17, 80-214 Gdańsk, Poland, email: mlgrzybowska@wp.pl (M.E.G.) \\ 3 Department of Physical Therapy, Medical University of Gdańsk, Dębinki 7, 80-211 Gdańsk, Poland, email: \\ erlabon@gumed.edu.pl (E.R.-L) \\ 4 Department of Physical Therapy, Medical University of Gdańsk, Dębinki 7, 80-211 Gdańsk, Poland; email: \\ rita.hansdorfer-korzon@gumed.edu.pl(R.H-K.) \\ 5 Department of Immunobiology and Environment Microbiology, Medical University of Gdańsk, Dębinki 7, \\ 80-211 Gdańsk, Poland, email: kzorena@gumed.edu.pl (K.Z.) \\ * Correspondence: zofia.barcikowska@gumed.edu.pl
}

\begin{abstract}
Introduction: In spite of many studies, the pathomechanism of dysmenorrhea (DM) remains unclear. The study aimed to evaluate the levels of progesterone and 17-beta estradiol and the severity of dysmenorrhea in six young women, including three after the use of manual therapy and the other three after the administration of ibuprofen. Material and methods: In six patients aged $22 \pm 2$, gynecological and physiotherapeutic examinations were completed. Moreover, a two-fold determination of progesterone and 17-beta estradiol levels were carried out. The intensity of dysmenorrhea was assessed in the numerical pain rating scale (NPRS). The patients were dichotomized into two subgroups. Subgroup A $(\mathrm{n}=3)$ was subjected to manual therapy $(3 \times 45 \mathrm{~min})$, while in subgroup B $(n=3)$, the patients were administered ibuprofen $3 \times 400 \mathrm{mg} / 24 \mathrm{~h}$ during menstruation. Results: Lower progesterone levels were detected in the blood of the 3 women from subgroup A after manual therapy and in 2 of the women from subgroup B after administrating ibuprofen. After the therapy, the level of 17-beta estradiol was higher in each woman from subgroup A and in one woman from subgroup B. In subgroup A, dysmenorrhea was reduced on average from 8 down to 3 points in the NPRS and duration time was shortened by one day on average. In subgroup B, dysmenorrhea was decreased on average from 8 to 3 points in the NPRS, yet no changes in its duration time were observed. Conclusions: It has been demonstrated that manual therapy alleviated dysmenorrhea to a similar degree as ibuprofen. However, only the manual therapy exerted an effect on the shortening of dysmenorrhea duration time. The use of manual therapy probably influenced the diminution of progesterone levels.
\end{abstract}

Keywords: dysmenorrhea; progesterone; manual therapy; non-steroidal anti-inflammatory drugs

\section{Introduction}

Despite numerous studies, the pathomechanism of dysmenorrhea is not fully understood. Previous studies have shown that dysmenorrhea is a complex process that may depend on many factors [1-4]. It is known that susceptibility to dysmenorrhea may be associated with genetic factors with the elevated secretion of prostaglandins and hormones such as estrogen and progesterone [4-6]. Our recent research has shown that women with dysmenorrhea were characterized by a family history of dysmenorrhea, the occurrence of premenstrual syndrome (PMS), early age of menarche, stressful lifestyle, 
lack of physical activity, and low self-esteem [7]. Management approaches for primary dysmenorrhea consist of pharmacological as well as non-pharmacological methods [8-11].

Pharmacological interventionsmay not be completely effectiveand have undesirable side effectsfor about $15 \%$ of females with primary dysmenorrhea [8]. Non-pharmacological interventions have been suggested for attaining relief from dysmenorrhea symptoms, including acupuncture and acupressure, biofeedback, heat management, transcutaneous electrical nerve stimulation, and relaxation techniques $[9,10]$. The use of manual therapy may be one of the additional forms of non-pharmacological support in the treatment of painful periods in young women.

Therefore, the aim of the research wasto evaluate the levels of progesterone and 17beta, estradiol, and the severity of dysmenorrhea in six young women, including three after the use of manual therapy and the other three after the administration of ibuprofen.

\section{Material and Methods}

A study was conducted in six women with dysmenorrhea, mean aged $22 \pm 2$ years. Recruitment was performed in 2019-2020. The women were qualified by gynecological and physiotherapeutic examination. The women enrolled in the study were divided into two equal subgroups.There were 3 women in subgroup A $(n=3)$ and 3 women in subgroup $B(n=3)$ with a mean age of 22 years and 21 years, respectively After qualification, during the first 3 days of menstruation from each participant were drawn blood samples to determine progesterone and 17-beta estradiol concentration. Subsequently, after menstruation in subgroup A, manual therapy was conductedonce a week by one menstrual cycle. Each woman underwent manual therapy 3 or 4 times (it depended on the menstrual cycle duration). One manual therapy session lasted 45 minutes. The manual therapy consists of techniques such as diaphragm stretching, manual release of pelvic floor muscles, poizometric relaxation of muscles, and trigger points manual therapy according to Simons and Travell of selected muscles. After manual therapy, during the next menstruation, a blood sample examination was repeated. Patients in subgroup A were instructed not to take painkillers prior to blood sampling. In women from subgroup B, manual therapy was not performed during the menstrual cycle. Scarcely, during the next menstruation did patients from subgroup B take ibuprofen in dose $3 \times 400 \mathrm{mg} / 24 \mathrm{~h}$. The blood samples were collected during ibuprofen action. Progesterone and 17-beta estradiol concentrations were measured using of Electrochemiluminescence (ECL) according to the manufacturer's protocol. Pain severity was assessed using the NumericPain Rating Scale (NPRS) [12]. The study was approved by the Ethics Committee of the Medical University of Gdańsk (no NKBBN/475/2018).The investigation was carried out following the principles of the Declaration of Helsinki as revised in 1996.

\section{Results}

The women from subgroup A were older (by 1 year on average) but with a lower mean BMI (by $3.0 \mathrm{~kg} / \mathrm{m}^{2}$ ) compared to women from subgroup B. In women from subgroup A, a lower level of progesterone was detected after manual therapy compared to baseline (mean 0.41 vs. $0.30 \mathrm{ng} / \mathrm{ml}$, respectively). However, the level of 17-beta estradiol was higher in each woman from subgroup A. The mean 17-beta estradiol levels increased in subgroup A from 28.0 to $36.0 \mathrm{pg} / \mathrm{ml}$ after manual therapy. In subgroup B, after administering ibuprofen, the level of progesterone decreased in 2 women from the mean value of $0.31 \mathrm{ng} / \mathrm{ml}$ before the therapy to $0.27 \mathrm{ng} / \mathrm{ml}$ after therapy. Additionally, the average concentration of 17-beta estradiol decreased from 27.0 to $17.0 \mathrm{pg} / \mathrm{ml}$. In one woman from subgroup B level of 17-beta estradiol was higher after having taken ibuprofen. In subgroup A, after manual therapy, the severity of dysmenorrhea was reduced on average from 8 down to 3 points in the NPRS scale and dysmenorrhea was shortened by one day on average (from 2 to 1 day). The use of ibuprofen in women from subgroup B decreased dysmenorrhea on average from 8 to 3 points in the NPRS but did notaffect dysmenorrhea duration.Table 1 presents the characteristics of the examined women with dysmenorrhea. 
Table 1. Characteristic of young women with dysmenorrhea.

\begin{tabular}{|c|c|c|c|c|}
\hline & \multicolumn{4}{|c|}{ Women with dysmenorrhea } \\
\hline & \multicolumn{2}{|c|}{ Subgroup A } & \multicolumn{2}{|c|}{ Subgroup B } \\
\hline Age (years) & \multicolumn{2}{|c|}{22.0} & \multicolumn{2}{|c|}{21.0} \\
\hline Body Mass Index (BMI) kg/m2 & \multicolumn{2}{|c|}{21.0} & \multicolumn{2}{|c|}{24.0} \\
\hline Manual therapy ( $3 \times 45 \mathrm{~min}$ ) & \multicolumn{2}{|c|}{ YES } & \multicolumn{2}{|c|}{$\mathrm{NO}$} \\
\hline Administered ibuprofen $3 \times 400 \mathrm{mg} / 24 \mathrm{~h}$ & \multicolumn{2}{|c|}{$\mathrm{NO}$} & \multicolumn{2}{|c|}{ YES } \\
\hline & Pre (mean) & Post(mean) & Pre (mean) & Post(mean) \\
\hline Progesterone $(\mathrm{ng} / \mathrm{ml})$ & 0.41 & 0.30 & 0.31 & 0.27 \\
\hline 17-beta estradiol (pg/ml) & 28.0 & 36.0 & 27.0 & 17.0 \\
\hline Numerical pain rating scale (NPRS) & 8 & 3 & 8 & 3 \\
\hline Duration of dysmenorrhea (days) & 2 & 1 & 3 & 3 \\
\hline
\end{tabular}

\section{Discussion}

This study evaluated the effectiveness of manual therapy in comparison to the use of ibuprofen on the severity of dysmenorrhea and their impact on the level of sex hormones. We demonstrated that manual therapy deceasesthe level of progesterone during menstruation among women with dysmenorrhea. According to our knowledge, this is the first study to show that manual therapy could have an impact on the level of progesterone. It was only suggested that manual therapy could decrease the level of progesterone among women with dysmenorrhea. One of the analyzed sex hormones was 17-betaestradiol. The average level of 17-beta estradiol was higher after the manual therapy, but lower during taking ibuprofen. One study provided data which evaluated the influence of acupuncture on the level of estradiol. Chinese researchers examined the effectiveness of acupuncture on hot flushes and estradiol levels among perimenopausal women. They demonstrated that acupuncture increases the secretion of estradiol [11]. The next analyzed in our study parameter was the duration of dysmenorrhea. Among women from subgroup A, the duration of dysmenorrhea was reducedafter manual therapy. Whereas, in subgroup $B$, no change was demonstrated. The reduction of dysmenorrhea duration corresponds with the study conducted by Schwerla et al. The authors showed that dysmenorrhea after osteopathic treatment lasted less than two days [13]. In the presented study, the intensity of dysmenorrhea was reduced among all surveyed women in subgroup A and subgroup B. The analgesic effect obtained was similar in both subgroups, independent of the kind of therapy. These results confirm earlier reports published by Barassi et al. [14]. The authors compared Neuromuscular Manual Therapy with pharmacological treatment. Their therapy reduced pain by about half on the NPRS scale. The effect of Neuromuscular Manual Therapy was comparable to the effect of pharmacological treatment.

\section{Conclusions}

The use of manual therapy in women could relieve dysmenorrhea to a similar degree as ibuprofen. However, only the manual therapy exerted an effect on the shortening of dysmenorrhea duration time. It is suggested that manual therapy probably influenced the diminution of the progesterone level. Further studies using a greater number of patients are required.

Author Contributions: Concept and design of the manuscript (Z.B., K.Z.), collection of literature (Z.B., K.Z.), original draft preparation (Z.B., K.Z.); project administration (M.E.G., E.R.-L., R.H.-K.); review and editing (M.E.G., E.R.-L., R.H.-K., K.Z.); editing and final approval (K.Z.). All authors read and approved the final manuscript.

Funding: This work was supported by the grant of Medical University of Gdańsk [ST-020108/07/780].

Conflicts of Interest: The authors declare no conflict of interest. 
1. Costantino C, Casuccio A, Restivo V.PotentialRisks and Factors of Women'sHealthPromotion. Int J Environ Res Public Health. 2020 Dec 21;17(24):9555. doi: 10.3390/ijerph17249555.

2. Iacovides, S.; Avidon, I.; Baker, F. C. What We Know about Primary Dysmenorrhea Today: A Critical Review. Hum. Reprod. Update2015, 21 (6), 762-778. https://doi.org/10.1093/humupd/dmv039.

3. Barcikowska, E. Rajkowska-Labon, M. E. Grzybowska, R. Hansdorfer-Korzon, and K. Zorena, Inflammatory markers in dysmenorrhea and therapeutic options. International Journal of Environmental Research and Public Health, vol. 17, no. 4, pp. 11911214, 2020. doi: 10.3390/ijerph17041191

4. Szmidt MK, Granda D, Sicinska E, Kaluza J.Primary Dysmenorrhea in Relation to Oxidative Stress and Antioxidant Status: A Systematic Review of Case-Control Studies. Antioxidants (Basel). 2020 Oct 15;9(10):994. doi: 10.3390/antiox9100994.

5. Jones A. V., Hockley J. R. F., Hyde C., et al. Genome-wide association analysis of pain severity in dysmenorrhea identifies association at chromosome 1p13.2, near the nerve growth factor locus. Pain. 2016;157(11):2571-2581. doi: 10.1097/j.pain.0000000000000678.

6. Haidari F, Homayouni F, Helli B, Haghighizadeh MH, Farahmandpour F. Effect of chlorella supplementation on systematic symptoms and serum levels of prostaglandins, inflammatory and oxidative markers in women with primary dysmenorrhea. Eur J ObstetGynecolReprod Biol. 2018 Oct;229:185-189. doi: 10.1016/j.ejogrb.2018.08.578.

7. Barcikowska, Z.; Wójcik-Bilkiewicz, K.; Sobierajska-Rek, A.; Grzybowska, M. E.; Wąż, P.; Zorena, K. Dysmenorrhea and Associated Factors among Polish Women: A Cross-Sectional Study. Pain Res. Manag.2020, 2020. https://doi.org/10.1155/2020/6161536.

8. Rizk, S. A. Effect of Aromatherapy Abdominal Massage using Peppermint Versus Ginger oils on Primary Dysmenorrhea among Adolescent Girls. Journal of American Science J Am Sci, 2013, 99(1111), 597-605

9. Ameade, E. P. K., Amalba, A., \& Mohammed, B. S. Prevalence of dysmenorrhea among University students in Northern Ghana; its impact and management strategies. BMC Women's Health,2018, 18(1), 39. https://doi.org/10.1186/s12905-018-0532-1

10. Xu D, Wang L, Deng L, Luo Y, Wei Y, Yan P.Intradermal acupuncture for primary dysmenorrhea: A protocol of systematic review and meta-analysis of randomized clinical trials. Medicine (Baltimore). 2020 Sep 11;99(37):e22188. doi: 10.1097/MD.0000000000022188

11. Cao, Z.; Tang, J.; Xue, Y.; Wang, Q.; Li, S.; Zhou, Y.; Zhang, W. Comparison between manual acupuncture and electroacupuncture for hot flashes and sex hormone of perimenopausal syndrome. Zhongguo Zhen Jiu2017, 37 (3), $247-252$. https://doi.org/10.13703/j.0255-2930.2017.03.007

12. Jensen MP, Turner JA, Romano JM, Fisher LD Comparative reliability and validity of chronic pain intensity measures. Pain . 1999; 83 (2): 157-162. doi: 10.1016 / s0304-3959 (99) 00101-3.

13. Schwerla, F.; Wirthwein, P.; Rütz, M.; Resch, K.-L. Osteopathic Treatment in Patients with Primary Dysmenorrhoea: A Randomised Controlled Trial. Int. J. Osteopath. Med.2014, 17 (4), 222-231. https://doi.org/10.1016/j.ijosm.2014.04.003.

14. Barassi, G.; Bellomo, R. G.; Porreca, A.; Di Felice, P. A.; Prosperi, L.; Saggini, R. Somato-Visceral Effects in the Treatment of Dysmenorrhea: Neuromuscular Manual Therapy and Standard Pharmacological Treatment. J. Altern. Complement. Med.2017, 00 (00), acm.2017.0182. https://doi.org/10.1089/acm.2017.0182. 